\title{
Thin-section Computed Tomography findings before and after azithromycin treatment of neutrophilic reversible lung allograft dysfunction
}

\author{
Pim A. de Jong • Robin Vos • Geert M. Verleden • \\ Bart M. Vanaudenaerde • Johny A. Verschakelen
}

Received: 6 April 2011 /Revised: 24 May 2011 / Accepted: 12 June 2011 /Published online: 27 July 2011

(C) The Author(s) 2011. This article is published with open access at Springerlink.com

\begin{abstract}
Objectives Recently a novel subgroup of bronchiolitis obliterans syndrome (BOS) has been described in patients after lung transplantation with high neutrophil counts in broncho-alveolar lavage and recovery of lung functional decline with azithromycin treatment. We aimed to describe the thin-section computed tomography (CT) findings of these neutrophilic reversible allograft dysfunction (NRAD) patients before and after azithromycin.

Methods A cohort of 100 lung transplant recipients with BOS were treated with azithromycin and underwent lung function testing, broncho-alveolar lavage and CT before azithromycin treatment and during follow-up. The $200 \mathrm{CT}$ data sets were scored for bronchial dilatation, mucus plugging, centrilobular abnormalities, airway wall thickening, consolidation, ground glass and end-expiratory air trapping.

Results NRAD was characterized by more centrilobular abnormalities on CT ( $p=0.03$ for prevalence and $p=0.06$ for severity) compared to non-responders. At follow-up NRAD patients showed improvement in all CT abnormalities including air trapping, but the degree of improvement in all CT abnormalities was significantly different between responders and non-responders (who showed progression of bronchus dilatation, consolidation and air trapping).
\end{abstract}

P. A. de Jong $\cdot$ J. A. Verschakelen

Department of Radiology, Katholieke Universiteit Leuven,

Leuven, Belgium

P. A. de Jong $(\bowtie) \cdot$ R. Vos $\cdot$ G. M. Verleden $\cdot$ B. M. Vanaudenaerde Lung Transplantation Unit, Katholieke Universiteit Leuven,

Leuven, Belgium

e-mail: pimdejong@gmail.com

P. A. de Jong

Department of Radiology, University Medical Center Utrecht,

Utrecht, The Netherlands
Conclusions Within BOS patients those with NRAD differ from azithromycin non-responders by more centrilobular abnormalities on CT before azithromycin and improvement in bronchus dilatation, consolidation and air trapping during treatment.

Keywords Computed tomography · Azithromycin . Bronchiolitis obliterans syndrome $\cdot$ Chronic allograft rejection $\cdot$ Neutrophilic broncho-alveolar lavage

\section{Key points}

1. Bronchiolitis obliterans syndrome was considered irreversible in chronic lung transplant rejection

2. Recently, neutrophilic reversible allograft dysfunction (NRAD), was recognized as a treatable form

3. Computed tomography can demonstrate specific abnormalities in these patients

4. Computed tomography may aid physicians in early and accurate diagnosis of NRAD

\section{Introduction}

The main obstacle to long-term survival of lung transplant recipients is the development of bronchiolitis obliterans syndrome (BOS) [1]. BOS is characterized by a progressive decline in the forced expiratory volume in one second $\left(\mathrm{FEV}_{1}\right)$ for which no explanation such as infection or bronchus stenosis can be found. The syndrome BOS is introduced to describe chronic allograft rejection in the absence of histological proof of obliterative bronchiolitis. Indeed, classically it is thought that the dysfunction is caused by an 
irreversible process called constrictive bronchiolitis, pathologically characterized by fibroproliferative damage to the small airways. The radiological feature of this fibroproliferative form of BOS has been described as extensive air trapping ( $>32 \%$ of the lung had to be involved) on end-expiratory CT in patients with normal inspiratory CT findings [2].

Important recent observations revealed that the graft dysfunction is not always irreversible, but there exists an important subgroup of BOS patients who respond to azithromycin treatment [3]. These patients typically have extensive neutrophils in the broncho-alveolar lavage fluid and this new entity was called neutrophilic reversible allograft/airways dysfunction (NRAD) [3]. NRAD is strictly defined as an increase in $\mathrm{FEV}_{1} \geq 10 \%$ after 3 to 6 months of azithromycin treatment (compared to $\mathrm{FEV}_{1}$ at start of treatment, based on two separate measurements with at least 3 weeks in between). Several groups [4-9] confirmed these observations and nowadays there is convincing evidence for the existence of NRAD $[5,9]$. However, the radiological findings have not been described systematically $[3,10]$. Knowledge about the presentation of NRAD on CT images would be important as not all patients respond to azithromycin and CT might be valuable in the diagnosis and treatment of this disease entity.

We aimed to describe the CT findings in patients with bronchiolitis obliterans syndrome either with or without neutrophilic reversible allograft dysfunction before and after azithromycin treatment.

\section{Methods}

\section{Subjects}

We studied a retrospective cohort of 100 lung transplant recipients that was selected in a single centre as follows. Between July 1991 and January 2009463 lung transplantations were performed. Patients who were never treated with a macrolide $(n=216)$, who were treated with a macrolide for other reasons than chronic lung allograft dysfunction $(n=56)$ or who participated in a placebo-controlled azithromycin trial $(n=83)$ were excluded. When the present study was designed, the trial was not unblinded, therefore these subjects were excluded. From the remaining 108 patients 1 was excluded because of follow-up less than 3 months and 7 were excluded because of missing CT data at the start of treatment and/or during follow-up. The resulting 100 patients were all treated with azithromycin for chronic lung allograft dysfunction. Treatment was following our clinical protocol starting with $250 \mathrm{mg}$ azithromycin per day for the first 5 days and subsequently $250 \mathrm{mg}$ azithromycin on Monday, Wednesday and Friday. The study was approved by the local University Hospital Ethical Review Board and all patients gave written informed consent.
CT protocol

The CT examinations were performed on a Siemens Somatom Sensation 16 or 64, a Siemens Definition Flash (Siemens AG, Erlangen Germany) or a Philips Brilliance 64 (Philips Medical Systems, Best, The Netherlands) without intravascular contrast media. One CT data set of the entire thorax was obtained in suspended deep inspiration in the supine position using $120 \mathrm{kV}$ and $140 \mathrm{mAs}$ and reconstructed as follows: $1 / 0.5 \mathrm{~mm}$ axial, $5 / 5 \mathrm{~mm}$ axial and 3/ $3 \mathrm{~mm}$ coronal displayed in lung and mediastinal windowcentre settings. Another $\mathrm{CT}$ data set was also obtained after breath-hold instruction at end- expiration with the patient supine but in a sequential mode with collimation $2 \times 1 \mathrm{~mm}$ and table feed of $30 \mathrm{~mm}$ using $120 \mathrm{kV}$ and $150 \mathrm{mAs}$. Reconstructions of $1 \mathrm{~mm}$ slice thickness were calculated and displayed in lung window-centre settings.

\section{CT scoring}

The resulting $200 \mathrm{CT}$ data sets in 100 patients were scored by using a previously described system [11]. This system semi-quantitatively scores on inspiratory $\mathrm{CT}$ the severity and extent of bronchus dilatation in the central and peripheral lung, extent of mucous plugging in large airways, extent of centrilobular nodules including tree-inbud, extent and severity of airway wall thickening in the central and peripheral lung, extent of consolidation and extent of ground glass opacities and extent of air trapping on expiratory CT. In general abnormalities were defined according to the Fleischner Society nomenclature [12]. More specifically, bronchus dilatation was defined as a bronchus lumen diameter greater than the accompanying pulmonary artery outer diameter, lack of tapering of the bronchus or bronchi visible in the outer centimeter of the lung. Airway wall thickening was defined as a wall thickness to artery diameter ratio $>0.2$, this was assessed subjectively [13]. Each abnormality was scored in six lung lobes (lingula included), and per lobe the extent involved with the abnormality was estimated as less than one-third, between one-third and two-thirds, and more than two-thirds of the lobar volume. All scores presented are expressed on a scale $0-100$ as previously described because this scale may enable easier interpretation. For example when one of six lobes demonstrated centrilobular changes and that lobe was involved with this abnormality for more than two-thirds, the centrilobular score would be $100 *((0+0+0+0+0+2) / 18)=$ $11 \%$, with 18 being the maximum possible score for that item. The CT score can thus be interpreted as a percentage of the lung being involved. CT data sets were scored by one observer for whom the reproducibility has previously been described [11]. CT examinations were scored independently in random order, but the observer was not blinded to 
Table 1 Characteristics of 100 lung transplant recipients with allograft dysfunction who were treated with azithromycin

\begin{tabular}{|c|c|c|c|}
\hline & $\begin{array}{l}\text { Patients responding to } \\
\text { azithromycin (NRAD) } \\
\mathrm{N}=41\end{array}$ & $\begin{array}{l}\text { Patients not responding } \\
\text { to azithromycin } \\
\mathrm{N}=59\end{array}$ & $\begin{array}{l}\text { Difference } \\
\text { between groups } \\
p \text {-value }\end{array}$ \\
\hline Age in years; Median $(25 \%-75 \%)$ & $52.0(40.5-59.0)$ & $49.0(29.0-56.0)$ & 0.30 \\
\hline Gender male; N (\%) & $21(52)$ & $31(53)$ & 0.90 \\
\hline Single lung transplant; N (\%) & $12(29)$ & $20(34)$ & 0.63 \\
\hline $\begin{array}{l}\text { Years after lung transplant of start allograft dysfunction; Median } \\
\text { (interquartile range) }\end{array}$ & $1.81(0.59-3.82)$ & $3.03(1.33-5.19)$ & 0.09 \\
\hline Months between CT Median (interquartile range) & $7.0(4.6-11.5)$ & $8.1(4.8-11.9)$ & 0.39 \\
\hline Neutrophils\% in broncho-alveolar lavage; Median (interquartile range) & $29.3(10.4-69.2)$ & $12.8(3.0-44.0)$ & 0.04 \\
\hline Neutrophils\% after azithromycin; Median (interquartile range) & $6.0(1.9-46.5)$ & $10.4(2.8-44.2)$ & 0.37 \\
\hline $\mathrm{FEV}_{1} \%$ before treatment; Median (interquartile range) & $67.0(52.0-82.5)$ & $60.0(46.0-76.0)$ & 0.10 \\
\hline $\mathrm{FEV}_{1} \%$ at follow-up; Median (interquartile range) & $82.0(64.5-99.0)$ & $52.0(38.0-73.0)$ & $<0.0001$ \\
\hline
\end{tabular}

$\mathrm{N}=$ Number, $\mathrm{FEV}_{1}=$ Forced expiratory volume in one second. Between group differences are tested with Mann-Whitney- $U$ test for continuous variables and Chi-square statistics for categorical variables. NRAD: neutrophilic reversible allograft dysfunction. Neutrophil counts were obtained at baseline in $34(83 \%)$ responders and $47(80 \%)$ non-responders and at follow-up in $29(71 \%)$ responders and 43 (73\%) non-responders

baseline or post-treatment status. The observer was blinded for patient characteristics including neutrophil count and azithromycin response. Both coronal and axial images could be used for scoring.

\section{Other investigations}

Broncho-alveolar lavage (BAL) was routinely performed, if possible, around fixed time points after lung transplantation (21 days, 3, 6, 12, 18, 24, 30 months, 3, 4, 5 years), or if acute rejection, infection or BOS was suspected. BAL fluid was evaluated for neutrophil\% and was cultured for pathogenic organisms. Neutrophil counts were obtained at baseline in 34 (83\%) responders and $47(80 \%)$ non-responders and at followup in $29(71 \%)$ responders and $43(73 \%)$ non-responders. Spirometry was performed in agreement with American
Thoracic Society-criteria, prior to bronchoscopy (Masterscreen, Jaeger, Germany) [14]. BOS was diagnosed and staged according to the The International Society for Heart and Lung Transplantation (ISHLT)-working formulation [1]. Response to azithromycin was defined as an increase in $\mathrm{FEV}_{1}$ $\geq 10 \%$ after 3 to 6 months of treatment (compared to $\mathrm{FEV}_{1}$ at start of treatment, based on two separate measurements with at least 3 weeks in between) and non-responders were defined as having an increase of less than $10 \%$.

Data analysis

Responders were compared to non-responders by using Mann-Whitney- $U$ test for continuous variables and Chisquare statistics for categorical variables. SPSS 18.0 (Inc. Chicago, IL, USA) was used for data analysis. Improve-

Table 2 Prevalence of thin-section CT findings in lung transplant recipients with allograft dysfunction before start of azithromycin treatment

\begin{tabular}{|c|c|c|c|}
\hline & $\begin{array}{l}\text { Patients responding } \\
\text { to azithromycin } \\
\text { (NRAD) } \\
N=41\end{array}$ & $\begin{array}{l}\text { Patients not responding } \\
\text { to azithromycin } \\
N=59\end{array}$ & $\begin{array}{l}\text { Difference between } \\
\text { groups } \\
p \text {-value }\end{array}$ \\
\hline Bronchus dilatation N (\%) & $13(32)$ & $25(42)$ & 0.28 \\
\hline Large airway mucus N (\%) & $10(24)$ & $13(22)$ & 0.78 \\
\hline Centrilobular nodules N (\%) & $22(54)$ & $19(32)$ & 0.03 \\
\hline Airway wall thickening N (\%) & $14(34)$ & $16(27)$ & 0.45 \\
\hline Consolidation N (\%) & $15(37)$ & $17(29)$ & 0.41 \\
\hline Ground glass N (\%) & $17(42)$ & $20(34)$ & 0.44 \\
\hline Air trapping $\mathrm{N}(\%)$ & $34(83)$ & $53(90)$ & 0.31 \\
\hline Air trapping $>32 \% \mathrm{~N}(\%)$ & $16(39)$ & $26(44)$ & 0.62 \\
\hline
\end{tabular}

Between group differences are tested with a Chi-square test; $N=$ number. NRAD: neutrophilic reversible allograft dysfunction 
Table 3 Thin-section CT scores in lung transplant recipients with allograft dysfunction before start of azithromycin treatment

\begin{tabular}{lccc}
\hline & $\begin{array}{l}\text { Patients responding } \\
\text { to azithromycin (NRAD) } \\
N=41\end{array}$ & $\begin{array}{l}\text { Patients not responding } \\
\text { to azithromycin } \\
N=59\end{array}$ & $\begin{array}{l}\text { Difference between } \\
\text { groups } \\
p \text {-value }\end{array}$ \\
\hline Bronchus dilatation; Mean (range) & $2.6(0.0-35.2)$ & $3.1(0.0-22.2)$ & 0.27 \\
Large airway mucus; Mean (range) & $3.7(0.0-27.8)$ & $3.2(0.0-33.3)$ & 0.73 \\
Centrilobular nodules; Mean (range) & $14.0(0.0-88.9)$ & $9.1(0.0-66.7)$ & 0.06 \\
Airway wall thickening; Mean (range) & $1.9(0.0-14.8)$ & $4.1(0.0-16.7)$ & 0.58 \\
Consolidation; Mean (range) & $3.9(0.0-44.4)$ & $7.1(0.0-66.7)$ & 0.55 \\
Ground glass; Mean (range) & $5.8(0.0-33.3)$ & $29.5(0.0-100.0)$ & 0.66 \\
Air trapping; Mean (range) & $22.6(0.0-55.6)$ & 0.27 \\
\hline
\end{tabular}

Between group differences are tested with Mann-Whitney- $U$ test. NRAD: neutrophilic reversible allograft dysfunction

ment and worsening in CT scores was defined as the posttreatment score being, respectively, lower and higher when compared to the pre-treatment CT score. Data are given as median (interquartile range) unless indicated otherwise and significance level was set at $P<0.05$.

\section{Results}

Clinical characteristics of responders (NRAD) versus non responders

Of the 100 subjects 41 responded and 59 did not respond to azithromycin treatment. Both responders and nonresponders were on average around 50 years of age, a little over $50 \%$ was male and approximately one-third received single lung transplantation. Clinical characteristics were not different between the groups, except the expected neutrophil percentage in the BAL before treatment which was significantly higher in the responders and the $\mathrm{FEV}_{1}$ after treatment which was significantly higher in the responders. These findings are summarized in Table 1.

Prevalence and severity of CT abnormalities before azithromycin

Before treatment NRAD responders had more often centrilobular changes (nodules and tree-in-bud, $p=0.03$ ) and tended to have higher centrilobular scores $(p=0.06)$ in comparison with non-responders. Bronchus dilatation, mucous plugging, airway wall thickening, consolidation, ground glass and air trapping presence and severity were not different between responders and non-responders at baseline. These findings are summarized in Tables 2 and 3 .

\section{Changes in CT findings after treatment}

In azithromycin responders all abnormalities including bronchus dilatation, mucous plugging, centrilobular abnormalities airway wall thickening, consolidation,

Table 4 Longitudinal thin-section CT scores in lung transplant recipients with allograft dysfunction during azithromycin treatment

\begin{tabular}{lccc}
\hline & $\begin{array}{l}\text { Patients responding } \\
\text { to azithromycin (NRAD) } \\
\text { Number=41 }\end{array}$ & $\begin{array}{l}\text { Patients not responding } \\
\text { to azithromycin } \\
\text { Number=59 }\end{array}$ & $\begin{array}{l}\text { Difference between } \\
\text { groups } \\
p \text {-value }\end{array}$ \\
\hline Bronchus dilatation; Mean (range) & $-0.79(-20.4-0.0)$ & $1.5(0.0-27.8)$ & $<0.0001$ \\
Large airway mucus; Mean (range) & $-2.4(-27.8-5.6)$ & $-0.09(-33.3-22.2)$ & 0.04 \\
Centrilobular nodules; Mean (range) & $-10.7(-88.9-22.2)$ & $-2.8(-55.6-22.2)$ & 0.001 \\
Airway wall thickening; Mean (range) & $-1.5(-14.8-3.7)$ & $-1.2(-12.0-4.6)$ & 0.004 \\
Consolidation; Mean (range) & $-3.0(-33.3-0.0)$ & $-0.28(-33.3-55.6)$ & 0.004 \\
Ground glass; Mean (range) & $-4.9(-33.3-0.0)$ & $3.5(-33.3-55.6)$ & 0.009 \\
Air trapping; Mean (range) & $-2.0(-33.3-38.9)$ & 0.02 \\
\hline
\end{tabular}

Between group differences are tested with Mann-Whitney- $U$ Test. NRAD: neutrophilic reversible allograft dysfunction 


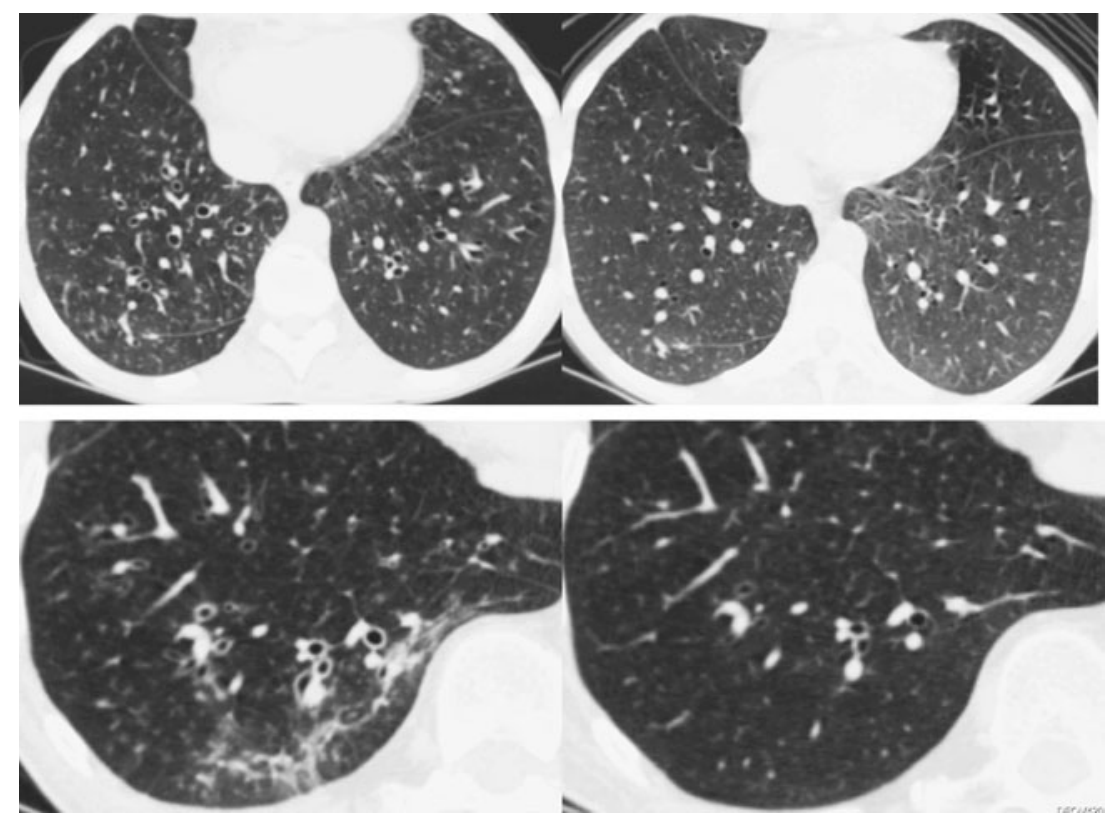

Fig. 1 Longitudinal CT findings in a 15-years old heart-lung transplant recipient with BOS responding to azythromycin (neutrophilic reversible allograft dysfunction (NRAD)). Four axial thin-slice CT images at inspiration before (left) and after (right) treatment with azithromycin in a patient who responded favorably. The pre-treatment $\mathrm{CT}$ images on the left demonstrate bronchial dilatation, bronchial wall thickening and centrilobular abnormalities all of which resolved on the follow-up CT images on the right. The lung allograft dysfunction occurred 3.2 years after transplantation, neutrophil counts are unknown. $\mathrm{FEV}_{1}$ had deteriorated $16 \%$ from baseline at the start of treatment and $13 \%$ was recovered after 6 months. This patient was judged to have reversible allograft dysfunction ground glass and air trapping improved significantly. The degree of improvement was for all CT abnormalities significantly different from the degree of change in the non-responders. In the non-responders some abnormalities improved (mucous plugging, centrilobular abnormalities, airway wall thickening and ground glass opacities), but bronchial dilatation, consolidation and air trapping worsened. These findings are summarized in Table 4. Figures 1, 2, 3 and 4 demonstrate the improvement in bronchial dilatation, centrilobular abnormalities, consolidation and air trapping in azithromycin responders. Figures 5 and 6 demonstrate progressive air trapping in non-responders with the classical fibroproliferative form of BOS or constrictive bronchiolitis.

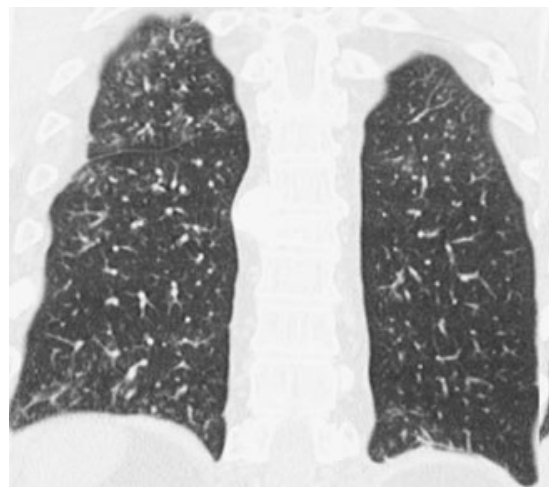

Fig. 2 Longitudinal CT findings in a 52-years old double lung transplant recipient with with BOS responding to azythromycin (neutrophilic reversible allograft dysfunction (NRAD)). Two coronal thin-slice CT imaging at inspiration before (left) and after (right) treatment with azithromycin in a patient who did respond. The pretreatment CT image demonstrates centrilobular nodules and tree-in-

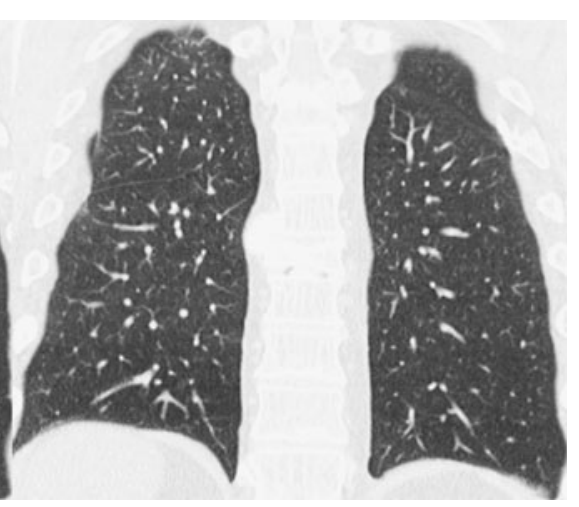

bud mainly at the apex and base of the right lung, which normalised on the follow CT. The lung allograft dysfunction occurred 4.2 years after transplantation, the broncho-alveolar lavage fluid revealed $89.6 \%$ neutrophils. $\mathrm{FEV}_{1}$ had declined to $75 \%$ at the start of treatment and improved to $90 \%$ in the subsequent 6 months. This patient was judged to have neutrophilic reversible allograft dysfunction 

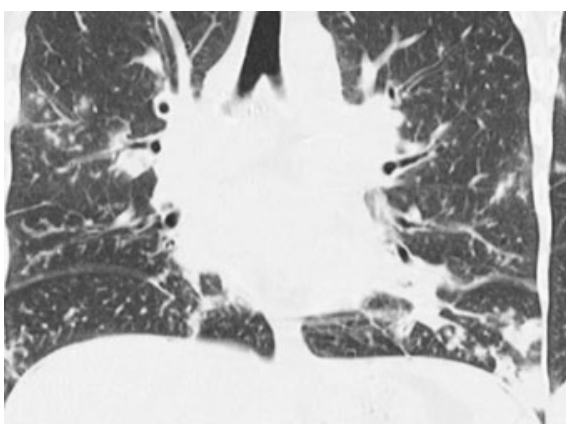

Fig. 3 Longitudinal CT findings in a 14-years old double lung transplant recipient with BOS responding to azythromycin (neutrophilic reversible allograft dysfunction (NRAD)). Two coronal thinslice CT imaging at inspiration before (left) and after (right) treatment with azithromycin in a patient who did respond. The pre-treatment CT image demonstrates centrilobular nodules and tree-in-bud and consol-

\section{Discussion}

We studied the thin-section CT imaging features in a cohort of lung transplant recipients with bronchiolitis obliterans syndrome (BOS) before and after azithromycin treatment. The BOS patients who did improve - those with neutrophilic reversible allograft dysfunction or NRAD — had significantly more often centrilobular abnormalities with or without tree-inbud on the pre-treatment CT. In this group the severity of bronchus dilatation, mucous plugging, centrilobular abnormalities, airway wall thickening, consolidation, ground glass and air trapping improved significantly more when compared with the BOS patients who did not improve.

The main obstacle to long-term survival of lung transplant recipients remains the development of bronchio-

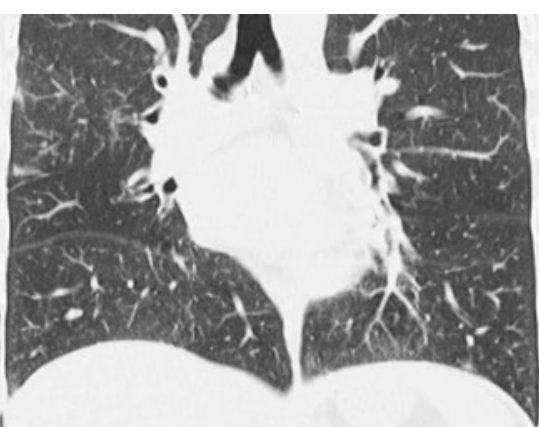

idation bilateral, which nearly reversed on the follow CT. The lung allograft dysfunction occurred 0.96 years after transplantation, the broncho-alveolar lavage fluid revealed $97 \%$ neutrophils. $\mathrm{FEV}_{1}$ had deteriorated $14 \%$ from baseline at the start of treatment and recovered in the subsequent 6 months. This patient was judged to have neutrophilic reversible allograft dysfunction

litis obliterans syndrome (BOS). Imaging research has focused on the thin-section CT features of this syndrome and whether these features may be able to demonstrate the syndrome earlier, before lung function deteriorates. It is indeed thought that only at a very early stage the process of obliterative bronchiolitis might be halted with intensive immune-suppression. In general, it is accepted that, although a variety of abnormalities are seen, air trapping is the main finding in patients with obliterative bronchiolitis [15-23] and air trapping involving more than $32 \%$ of the lung was shown to have good accuracy for the diagnosis of BOS [2]. Whether thin-section CT in lung transplant recipients is able to detect disease earlier than spirometry or transbronchial biopsy remains controversial, with some studies suggesting its value [2,24], while others could not
Fig. 4 Longitudinal CT findings in two lung transplant recipients with BOS responding to azythromycin (neutrophilic reversible allograft dysfunction (NRAD)) who demonstrated a reduction in the extent of air trapping on expiratory CT. Axial thin-slice CT images at end-expiration before (left) and after (right) treatment with azithromycin in two patients (upper and lower panel) who did respond to azithromycin. Air trapping decreased to some extent on $\mathrm{CT}$ which is inconsistent with fibroproliferative obliterative bronchiolitis as this is thought to be an irreversible process

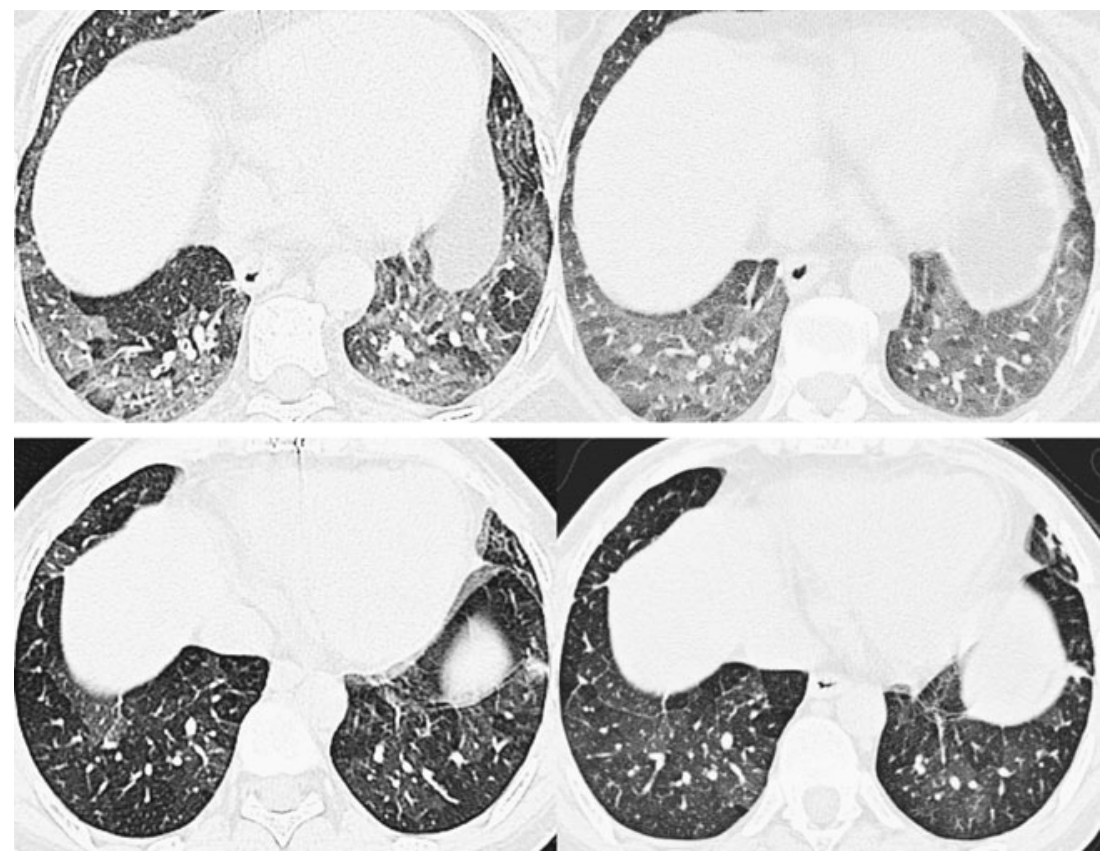




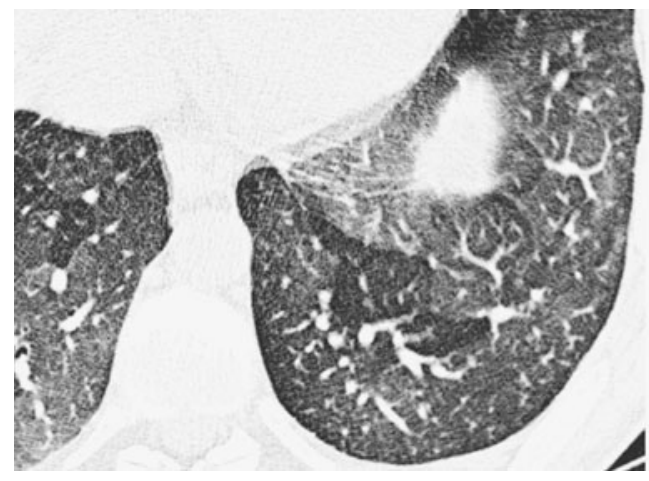

Fig. 5 Longitudinal CT findings in a 33-years old double lung transplant recipient with progressive air trapping whose allograft dysfunction did not respond to azithromycin therapy. Two axial thinslice CT imaging at end-expiration before (left) and after (right) treatment with azithromycin in a patient who did not respond. The pre-treatment CT image demonstrates some secondary pulmonary lobules with air trapping. The follow-up CT image on the right

confirm these findings [18-20, 23]. Interestingly the paradigm has changed for an important subgroup of BOS patients as this 'irreversible' disease has proven reversible in a substantial subgroup with azithromycin therapy. Azithromycin is a macrolide antibiotic which has antibacterial but also a variety of immunomodulatory effects [25]. These patients are further characterized by extensive neutrophils in the broncho-alveolar lavage fluid (without specific pathogens being cultured) and research to determine the pathogenesis is actively ongoing [26]. In order to determine whether thin-section CT could have a diagnostic

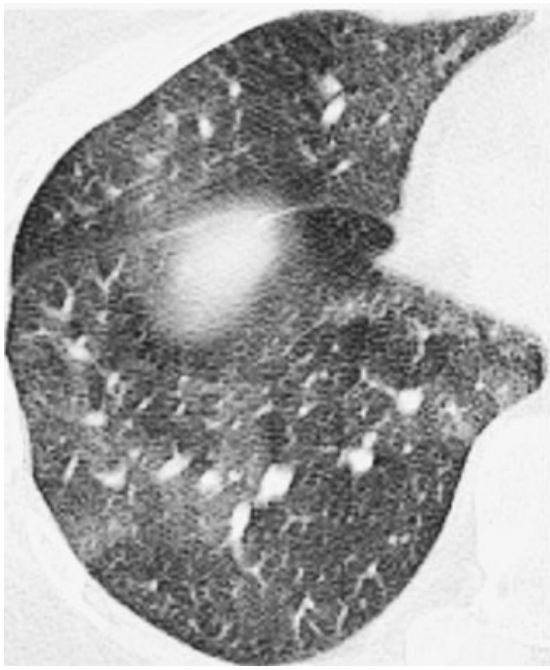

Fig. 6 Longitudinal CT findings in a 29-years old single right lung transplant recipient with subtle progressive air trapping whose dysfunction did not respond to azithromycin therapy. Two axial thin-slice CT images at end-expiration before (left) and after (right) treatment with azithromycin in a patient who did not respond. The pre-treatment CT image demonstrates about half of the secondary pulmonary lobules with air trapping. The follow-up CT image on the right demonstrates more

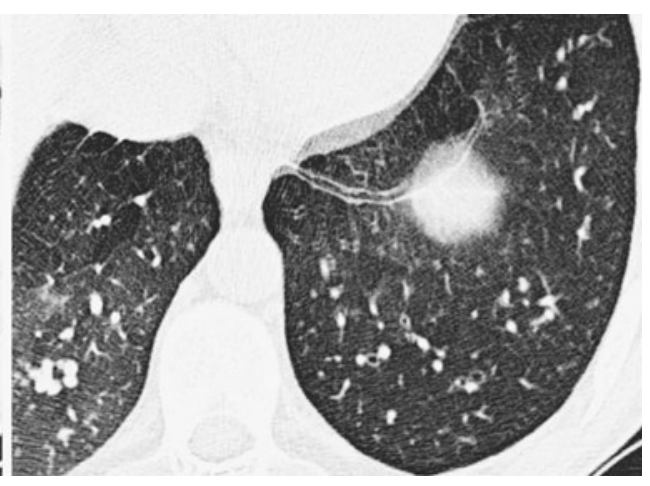

demonstrates more lobules with air trapping in the medial right lower lobe and diffuse air trapping in the left lower lobe. The lung allograft dysfunction occurred 1.4 years after transplantation, the bronchoalveolar lavage fluid revealed $3.5 \%$ neutrophils. $\mathrm{FEV}_{1}$ had declined to $76 \%$ at the start of treatment and declined further to $46 \%$ in the subsequent 6 months. This patient was judged to have the original form of obliterative bronchiolitis

role in this new disorder, the signs have to be known first. Therefore the present study is important since we showed that most CT abnormalities largely overlap between NRAD patients and non-responders to azithromycin, but that centrilobular nodules including tree-in-bud are significantly more common in NRAD patients. For the non-responders the question still remains whether CT has a role in the early diagnosis of small airways narrowing in the fibrotic subgroup of bronchiolitis obliterans syndrome.

We also showed the changes in CT abnormalities under azithromycin treatment. Especially the improvement in air

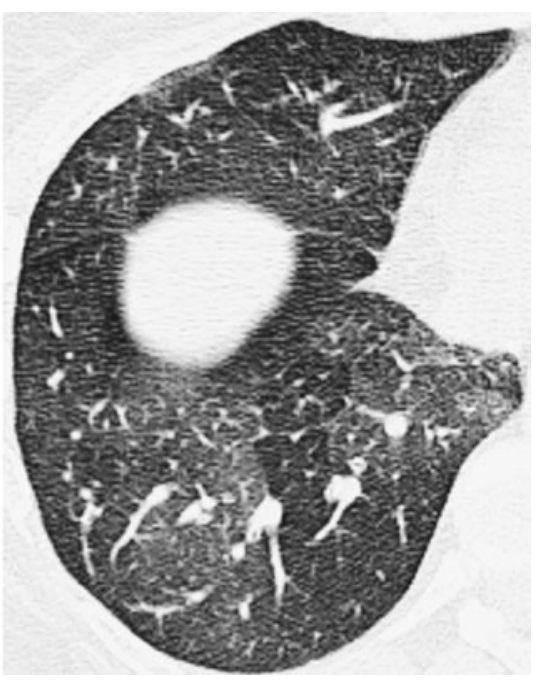

lobules lateral in the lower lobe and more diffuse air trapping in the middle lobe. The lung allograft dysfunction occurred 2.8 years after transplantation, the broncho-alveolar lavage fluid revealed $6.0 \%$ neutrophils. $\mathrm{FEV}_{1}$ had declined to $40 \%$ at the start of treatment and declined further to $30 \%$ in the subsequent 6 months. This patient was judged to have the fibrotic subgroup of bronchiolitis obliterans syndrome, probably representing true obliterative bronchiolitis 
trapping is interesting as this challenges the conventional opinion that air trapping is due to irreversible constrictive bronchiolitis in all patients with bronchiolitis obliterans syndrome. Apparently the air trapping in NRAD patients cannot be fully explained by obliterative bronchiolitis and possibly spasm, plugging or inflammation of the walls of small airways may be responsible for the reversible air trapping. Also bronchial dilatation was reversible in some patients and the dilated bronchi on pre-treatment $\mathrm{CT}$ does not necessarily represent irreversible bronchiectasis in lung transplant recipients with NRAD [10]. This 'reversible bronchiectasis' phenomenon has also been observed by others in acute disease [27, 28].

Our study has limitations. First, we did not control for inspiratory or expiratory levels, other than using breathhold instruction by experienced CT technicians. Air trapping can be masked by insufficient expiration. We therefore re-evaluated the subjects with improvement in air trapping scores, which convinced us that air trapping can be reversible in NRAD subjects, which is not explained by level of expiration or other factors. Second, we used a single reader in this study, given the time-consuming nature of detailed visual scores and the previously described observer agreement in a different cohort of lung transplant recipients [11]. The presented findings are fully in line with our clinical impression and we checked the CT scores of the subjects with 'reversible' air trapping, therefore we have no doubts about our findings.

In conclusion, post lung transplant patients with bronchiolitis obliterans syndrome showing recovery of the lung functional decline with azithromycin treatment (neutrophilic reversible lung allograft dysfunction (NRAD)) differ from patients showing no recovery by more centrilobular abnormalities on thin-section CT before the start of treatment and by regression of bronchus dilatation, consolidation and air trapping that are not seen in non-responders. The optimal diagnostic strategy for NRAD and the role of CT imaging, especially centrilobular disease, and neutrophil counts requires further research. Radiologists could include neutrophilic reversible allograft dysfunction (NRAD) in the differential diagnosis of lung transplant recipients with allograft dysfunction when centrilobular abnormalities with or without a tree-in-bud pattern are seen on thin-section CT.

Acknowledgement Research Foundation-Flanders (FWO: G.0723.10, G.0643.08). BMV is a senior research fellow of the FWO. GMV is holder of the GSK Chair in Respiratory Pharmacology funded by the Katholieke Universiteit Leuven: OT10/050, Belgium.

Open Access This article is distributed under the terms of the Creative Commons Attribution Noncommercial License which permits any noncommercial use, distribution, and reproduction in any medium, provided the original author(s) and source are credited.

\section{References}

1. Estenne M, Maurer JR, Boehler A, Egan JJ, Frost A, Hertz M, Mallory GB, Snell GI, Yousem S (2002) Bronchiolitis obliterans syndrome 2001: an update of the diagnostic criteria. J Heart Lung Transplant 21:297-310

2. Bankier AA, Van Muylem A, Knoop C, Estenne M, Gevenois PA (2001) Bronchiolitis obliterans syndrome in heart-lung transplant recipients: diagnosis with expiratory CT. Radiology 218:533-539

3. Vanaudenaerde BM, Meyts I, Vos R, Geudens N, De Wever W, Verbeken EK, Van Raemdonck DE, Dupont LJ, Verleden GM (2008) A dichotomy in bronchiolitis obliterans syndrome after lung transplantation revealed by azithromycin therapy. Eur Respir J 32:832-843

4. Gerhardt SG, McDyer JF, Girgis RE, Conte JV, Yang SC, Orens JB (2003) Maintenance azithromycin therapy for bronchiolitis obliterans syndrome: results of a pilot study. Am J Respir Crit Care Med 168:121-125

5. Verleden GM, Dupont LJ (2004) Azithromycin therapy for patients with bronchiolitis obliterans syndrome after lung transplantation. Transplantation 77:1465-1467

6. Yates B, Murphy DM, Forrest IA, Ward C, Rutherford RM, Fisher AJ, Lordan JL, Dark JH, Corris PA (2005) Azithromycin reverses airflow obstruction in established bronchiolitis obliterans syndrome. Am J Respir Crit Care Med 172:772-775

7. Shitrit D, Bendayan D, Gidon S, Saute M, Bakal I, Kramer MR (2005) Long-term azithromycin use for treatment of bronchiolitis obliterans syndrome in lung transplant recipients. J Heart Lung Transplant 24:1440-1443

8. Gottlieb J, Szangolies J, Koehnlein T, Golpon H, Simon A, Welte T (2008) Long-term azithromycin for bronchiolitis obliterans syndrome after lung transplantation. Transplantation 85:36-41

9. Vos R, Vanaudenaerde BM, Ottevaere A, Verleden SE, De Vleeschauwer SI, Willems-Widyastuti A, Wauters S, Van Raemdonck DE, Nawrot TS, Dupont LJ, Verleden GM (2010) Long-term azithromycin therapy for bronchiolitis obliterans syndrome: divide and conquer? J Heart Lung Transplant 29:1358-68

10. Verleden GM, Dupont LJ, Vanhaecke J, Daenen W, Van Raemdonck DE (2005) Effect of azithromycin on bronchiectasis and pulmonary function in a heart-lung transplant patient with severe chronic allograft dysfunction: a case report. J Heart Lung Transplant 24:1155-1158

11. de Jong PA, Dodd JD, Coxson HO, Storness-Bliss C, Paré PD, Mayo JR, Levy RD (2006) Bronchiolitis obliterans following lung transplantation: early detection using computed tomographic scanning. Thorax 61:799-804

12. Hansell DM, Bankier AA, MacMahon H, McLoud TC, Müller NL, Remy J (2008) Fleischner Society: glossary of terms for thoracic imaging. Radiology 246:697-722

13. Matsuoka S, Uchiyama K, Shima H, Ueno N, Oish S, Nojiri Y (2003) Bronchoarterial ratio and bronchial wall thickness on highresolution $\mathrm{CT}$ in asymptomatic subjects: correlation with age and smoking. AJR Am J Roentgenol 180:513-518

14. American Thoracic Society (1995) Standardization of spirometry, 1994 update. Am J Respir Crit Care Med 152:1107-1136

15. Dodd JD, de Jong PA, Levy RD, Coxson HO, Mayo JR (2008) Conventional high-resolution CT versus contiguous multidetector $\mathrm{CT}$ in the detection of bronchiolitis obliterans syndrome in lung transplant recipients. J Thorac Imaging 23:235-243

16. Leung AN, Fisher K, Valentine V, Girgis RE, Berry GJ, Robbins RC, Theodore J (1998) Bronchiolitis obliterans after lung transplantation: detection using expiratory HRCT. Chest 113:365-370

17. Siegel MJ, Bhalla S, Gutierrez FR, Hildebolt C, Sweet S (2001) Postlung transplantation bronchiolitis obliterans syndrome: usefulness of expiratory thin-section CT for diagnosis. Radiology 220:455-462 
18. Lee ES, Gotway MB, Reddy GP, Golden JA, Keith FM, Webb WR (2000) Early bronchiolitis obliterans following lung transplantation: accuracy of expiratory thin-section $\mathrm{CT}$ for diagnosis. Radiology 216:472-477

19. Konen E, Gutierrez C, Chaparro C, Murray CP, Chung T, Crossin J, Hutcheon MA, Paul NS, Weisbrod GL (2004) Bronchiolitis obliterans syndrome in lung transplant recipients: can thin-section CT findings predict disease before its clinical appearance? Radiology 231:467-473

20. Berstad AE, Aaløkken TM, Kolbenstvedt A, Bjørtuft O (2006) Performance of long-term CT monitoring in diagnosing bronchiolitis obliterans after lung transplantation. Eur J Radiol 58:124-131

21. Knollmann FD, Kapell S, Lehmkuhl H, Schulz B, Böttcher H, Hetzer R, Felix R (2004) Dynamic high-resolution electron-beam CT scanning for the diagnosis of bronchiolitis obliterans syndrome after lung transplantation. Chest 126:447-456

22. Choi YW, Rossi SE, Palmer SM, DeLong D, Erasmus JJ, McAdams HP (2003) Bronchiolitis obliterans syndrome in lung transplant recipients: correlation of computed tomography findings with bronchiolitis obliterans syndrome stage. J Thorac Imaging 18:72-79

23. Miller WT Jr, Kotloff RM, Blumenthal NP, Aronchick JM, Gefter WB, Miller WT (2001) Utility of high resolution computed tomography in predicting bronchiolitis obliterans syndrome following lung transplantation: preliminary findings. J Thorac Imaging $16: 76-80$

24. Knollmann FD, Ewert R, Wündrich T, Hetzer R, Felix R (2002) Bronchiolitis obliterans syndrome in lung transplant recipients: use of spirometrically gated CT. Radiology 225: 655-662

25. Friedlander AL, Albert RK (2010) Chronic macrolide therapy in inflammatory airways diseases. Chest 138:1202-1212

26. Verleden GM, Vos R, De Vleeschauwer SI, Willems-Widyastuti A, Verleden SE, Dupont LJ, Van Raemdonck DE, Vanaudenaerde BM (2009) Obliterative bronchiolitis following lung transplantation: from old to new concepts? Transpl Int 22:771-779

27. Kim JS, Tanaka N, Newell JD, Degroote MA, Fulton K, Huitt G, Lynch DA (2005) Nontuberculous mycobacterial infection: CT scan findings, genotype, and treatment responsiveness. Chest 128:3863-3869

28. Gaillard EA, Carty H, Heaf D, Smyth RL (2003) Reversible bronchial dilatation in children: comparison of serial highresolution computer tomography scans of the lungs. Eur J Radiol 47:215-20 